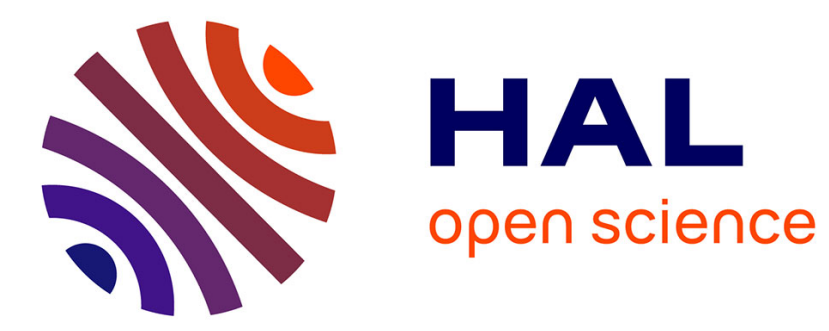

\title{
Particle identification with the ATLAS electromagnetic calorimeter
}

S. Tisserant

\section{To cite this version:}

S. Tisserant. Particle identification with the ATLAS electromagnetic calorimeter. TRDs for the Third Millenium - 3rd Workshop on Advanced Transition Radiation Detectors for Accelerators and Space Applications, Sep 2005, Brindisi, Italy. pp.321-325, 10.1016/j.nima.2006.02.151 in2p3-00105510

\section{HAL Id: in2p3-00105510 https://hal.in2p3.fr/in2p3-00105510}

Submitted on 13 Oct 2006

HAL is a multi-disciplinary open access archive for the deposit and dissemination of scientific research documents, whether they are published or not. The documents may come from teaching and research institutions in France or abroad, or from public or private research centers.
L'archive ouverte pluridisciplinaire HAL, est destinée au dépôt et à la diffusion de documents scientifiques de niveau recherche, publiés ou non, émanant des établissements d'enseignement et de recherche français ou étrangers, des laboratoires publics ou privés. 


\title{
Particle identification with the ATLAS electromagnetic calorimeter
}

\author{
Sylvain Tisserant ${ }^{*}$ \\ Centre de Physique des Particules de Marseille, Univ. Méditerranée, IN2P3-CNRS, F-13288 Marseille Cedex
}

Elsevier use only: Received date here; revised date here; accepted date here

\begin{abstract} separation) are summarized. (c) 2001 Elsevier Science. All rights reserved

Keywords : Calorimeter, Particle physics.

PACS: the PACS codes can be found at the home page of NIMA (left column, under Contents Services): http://www.elsevier.nl/inca/publications/store/5/0/5/7/0/1/
\end{abstract}

The design constraints and the target performances of the ATLAS electromagnetic calorimeter are reviewed. The construction and installation status is summarized. Some test-beam results, covering a large part of the final detector, are summarized. Some recent updates concerning the use of the electromagnetic calorimeter for particle identification $\left(\gamma / \pi^{0}\right.$, e $/ \pi$ and jet $/ \pi$

\section{Introduction}

The CERN Large Hadron Collider (LHC) is a proton-proton collider with $14 \mathrm{TeV}$ centre of mass energy and design luminosity of $10^{34} \mathrm{~cm}^{-2} \mathrm{~s}^{-1}$. The ATLAS (A Toroidal LHC Apparatus) detector is one of the two multi-purpose experiments under construction for the LHC, designed to maximize the discovery potential for new physics phenomena such as Higgs bosons and supersymmetric particles. It consists of three main parts. The inner tracking detectors, enclosed in a solenoid magnet in the central part, the calorimeters followed by a large air- core muon spectrometer. This one defines the global dimensions (diameter $22 \mathrm{~m}$, length $42 \mathrm{~m}$ ) of the detector. Excellent electromagnetic energy measurement and particle identification were two of the most important design criteria for the ATLAS experiment.

\section{Electromagnetic calorimeter design}

The ATLAS electromagnetic calorimeter obey to many constraints which have been determined from physics simulation [1]. In the following we summarize only a few of these constrains.

\footnotetext{
${ }^{*}$ Corresponding author. Tel.: +(33) 4918272 70; fax: +(33) 4918272 99; e-mail: tisserant@cppm.in2p3.fr.
} 
The hermeticity should be as perfect as possible and the design should avoid uninstrumented areas. For Higgs boson search through the decays to two photons or to four electrons, the calorimeter should allow $1 \%$ resolution on the Higgs mass measurement. This translates into a sampling term of $10 \% / \sqrt{\mathrm{E}}$ or better, and a constant term better than $1 \%$.

The dynamic range has to cover $30 \mathrm{MeV}$ up to $1 \mathrm{TeV}$, i.e. from the typical noise level up to the single cell energy deposit expected in the case of a $Z$ ' or W' boson with a mass of $6 \mathrm{TeV}$.

The detector has to be able to identify electrons with transverse momentum as low as $1-2 \mathrm{GeV} / \mathrm{c}$ up to a few TeV/c. The rejection factor against jets should be of the order of $10^{5}$ to select electrons exclusively, as a starting point for analysis using high $p_{T}$ leptons. To observe the $\mathrm{H} \rightarrow \gamma \gamma$ decay, a rejection factor of 3000 is needed to sufficiently suppress the $\gamma$-jet and jet-jet backgrounds. The ability to distinguish between $\pi^{0}$ and photons is essential to achieve this goal.

To address the above requirements, ATLAS has chosen to build a Lead/Liquid Argon sampling electromagnetic calorimeter, comprising a barrel and two end-cap parts. Liquid Argon technology has been chosen because of its intrinsic linear behavior as function of the deposited energy, stability of the response and radiation tolerance. A detailed description of the liquid argon calorimeters can be found in [2].

The barrel calorimeter, covering the $|\eta|$ range from 0 to 1.475 , shares its cryostat with the superconducting solenoid, the calorimeter being behind the solenoid. Each end-cap cryostat contains electromagnetic, hadronic and forward Liquid Argon calorimeters. In the electromagnetic end-cap calorimeter covers the $|\eta|$ range from 1.375 to 3.2.

Since the material in front of the electromagnetic calorimeter amounts to about $1.5 \mathrm{X}_{0}$ on average, up to $2 \mathrm{X}_{0}$ at the barrel to end-cap transition, the barrel and both end-cap calorimeters are complemented with presampler detectors that cover up to $|\eta|=1.8$. Their purpose is to evaluate the amount of energy loss in the inactive material in front of the calorimeter. Basically, these presamplers are thin layers of liquid argon equipped with readout electrodes but no absorbers.
The peculiar shape of the accordion has been chosen for the absorbers and electrodes of the liquid argon electromagnetic calorimeter because it allows to build the detector without any cracks in $\phi$, with the $\mathrm{HV}$ supplying cables and signal cables running on the front and back faces of the detector. This accordion geometry gives some $\phi$ response modulations, but its parameters can be fine-tuned to make these modulations very small : at the level of a few per mile. By reducing the length of the path needed to connect the readout cells to the readout electronics, the accordion shape allows to minimize the inductance in the signal path. Thus it is possible to use a fast shaping circuit to cope with the $25 \mathrm{~ns}$ bunch crossing time of the LHC.

The barrel calorimeter $\eta$ range is covered by two read-out electrodes, the first one extending from $|\eta|=0$ to $|\eta|=0.8$, and the second one covering the range from $|\eta|=0.8$ to $|\eta|=1.4$. The transition between these two electrodes corresponds to a change in lead thickness. This change is needed to prevent the sampling ratio of the calorimeter from increasing too much at high pseudo-rapidity.

The end-cap calorimeter has a mechanical structure similar to the barrel one, but with absorbers arranged like the spokes of a bicycle wheel. However, whereas the barrel calorimeter uses only one type of absorber (but using two lead thicknesses) and has a liquid argon gap thickness constant over the whole detector, in the end-cap calorimeter the accordion geometry and the gap thickness vary as function of $|\eta|$. Furthermore, the end-cap calorimeter uses two types of absorbers (different in shape and lead thickness), one for the inner area $(2.5<|\eta|<3.2)$ and one different type for the outer area $(1.4<|\eta|<2.5)$. By this way each end-cap calorimeter consists of two concentric wheels. The varying gap thickness requires different $\mathrm{HV}$ values as function of $\eta$ regions, in order to maintain an almost constant response with $\eta$. The structure and the understanding of the end-cap calorimeter is therefore more complex than in the case of the barrel structure. The end-cap calorimeter has also two types of electrodes corresponding to the inner and outer wheels.

The tracker detectors cover up to $|\eta|=2.5$, which is the region that will be used by ATLAS for "precision physics". Combining tracking and 
calorimeter information in this region requires the calorimeter to be fine segmented, in order to ensure an efficient tracking-calorimeter association. Above $|\eta|=2.5$ the calorimeter granularity is coarser.

In the barrel, the first compartment in depth has a very fine $\eta$ granularity $(\Delta \eta \times \Delta \phi=0.003 \times 0.1)$, in order to optimize the ability to separate photons from $\pi^{0}$ energy deposits, and electrons from jets. In the end-cap the width of the readout pads devoted to this first sampling are kept almost constant, this yields to an $\eta$ granularity varying as function of $\eta$ regions. The second compartment, devoted to energy measurement, has the same granularity of $\Delta \eta \times \Delta \phi=0.025 \times 0.025$ in both barrel and end-cap calorimeters. The third compartment has a slightly coarser granularity $\Delta \eta \times \Delta \phi=0.050 \times 0.025$.

\section{Performances}

The barrel is divided into two half barrels, each one containing 16 modules. Each end-cap calorimeter is divided into 8 modules. Four barrel and three endcap calorimeter modules have been tested in testbeam at CERN between 2000 and 2002. While the analysis is still ongoing, the already established results allow to have a good conviction of the quality of the detector. Among the various topics studied, we have now well established results on energy resolution, response uniformity, position resolution, and signal linearity.

\subsection{Energy resolution}

Energy resolution as function of electron energy is the first performance of the calorimeters that has been measured in test-beam [3][4]. For both barrel and end-cap modules, it has been found that the results are satisfactory with respect to the requirements and in good agreement with the simulation. For example, at $\eta=0.3625$ (in the barrel) the measured sampling term is $9.24 \%$ with a local constant term of $0.23 \%$. In the end-cap, at $\eta=1.9$, we have obtained a sampling term of $10.3 \%$ and a local constant term of $0.27 \%$.

\subsection{Uniformity of the detector response}

The uniformity of the detector response has been checked over the four barrel modules and three endcap modules. The uniformity is estimated as the r.m.s. of the distribution of the calorimeter response cell per cell. For the barrel the region around $\eta=0.8$, corresponding to the transition between the two types of electrodes and also to the lead thickness change, is still worked on. For most of the barrel module cells, the uniformity is $0.57 \%$. In the case of the end-cap modules, the uniformity on regions of a size of $\Delta \eta \times \Delta \phi=0.2 \times 0.4$ is of the order of $0.4 \%-0.6 \%$. On a complete module we obtain an uniformity of $0.6 \%$, yielding to a global constant term of $0.7 \%$.

These results demonstrate the excellent quality of the construction of both barrel and end-cap modules.

\subsection{Position and angular resolution}

A good shower position resolution is important for the Higgs mass measurement in the $\mathrm{H} \rightarrow \gamma \gamma$ decay channel. It has been measured in the barrel [5] and end-cap modules. Figure 1 shows the measured $\eta$ resolution for a barrel module as function $\eta$ for both the first and second compartments, compared with the simulation predictions. These measurements correspond to a position resolution of $\sim 250$ and $\sim 500 \mu \mathrm{m}$ in the first and second samplings respectively. The knowledge of the shower barycenters in both compartments allows an extraction of the vertex position and the incident direction of the shower. At $245 \mathrm{GeV}$, the vertex resolution varies as function of $\eta$ from $5 \mathrm{~mm}$ at $\eta=0$ to about $15 \mathrm{~mm}$ at $\eta=1$.

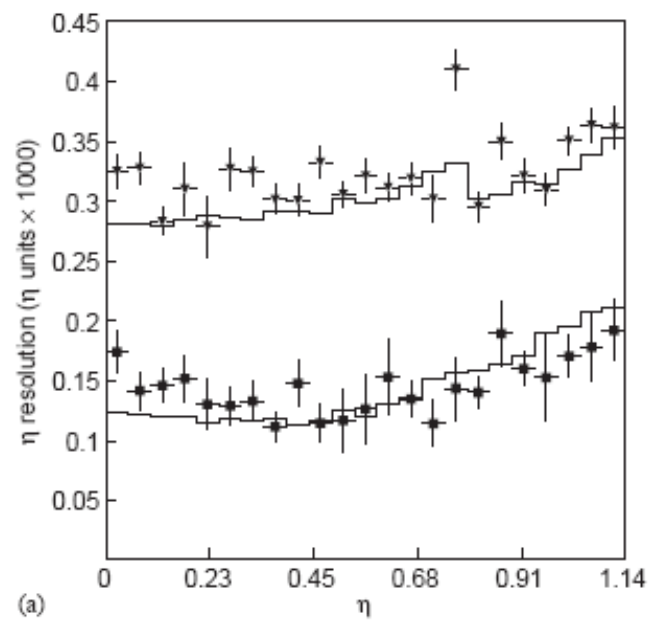

Fig. 1. $\eta$ resolution as function of $\eta$, at $\phi=0.26, \mathrm{E}=245 \mathrm{GeV}$ for the first (squares) and second (triangles) compartments of a barrel module, superimposed on the simulation prediction. 


\section{Status of the construction}

The barrel is divided into two half barrels, each one containing 16 modules. Each barrel modules consists of 64 absorbers and 128 electrodes. The construction of these series modules took about three years, including procurement, production of absorbers and electrodes as well as the stacking itself. It was finished in April 2003. Then the two half barrels were successfully assembled and inserted into the cryostat. After insertion, extensive electrical tests and measurements have been performed. The readout channels exhibiting problems represent less than 0.2 per mile of the 100000 channels. The barrel is in the ATLAS pit since fall 2004.

Each end-cap calorimeter consists of 8 modules. The construction, assembly and insertion of both endcap calorimeter was finished in fall 2004. Electrical tests have been performed on the first end-cap giving the similar results to the barrel ones. This first endcap should be installed down the ATLAS pit by the end of 2005.

\section{Photon/neutral pion separation}

The $\mathrm{H} \rightarrow \gamma \gamma$ decay mode is one of the most promising way to detect a Standard Model Higgs boson at the LHC in the $80-130 \mathrm{GeV} / \mathrm{c}^{2}$ mass range. After jet rejection, the surviving events consist mainly of isolated $\pi^{0}$ s. To reject these remaining events the calorimeter has to provide a rejection of about 3 for a photon identification efficiency of $90 \%$.

The expected performance was been evaluated by using single photons and single $\pi^{0}$, s, fully simulated through the inner detector and the electromagnetic calorimeter. A description of the separation principles can be found in ref. [6]. The $\gamma / \pi^{0}$ separation relies mainly on the fine granularity of the first compartment in the precision region used to analyze the shower shape. A continuous work is performed since many years in order to cope with experimental difficulties as the measured cross-talk between readout cells of the electromagnetic calorimeter and the photon conversion in the inner tracker as well. The cuts have been optimized to keep in presence of cross-talk the same performance level $\left(\pi^{0}\right.$ rejection and $\gamma$ efficiency) as obtained in [6]. The photon conversion can deteriorate the rejection factor by about $25 \%$. As we observe a strong correlation between the position of the conversion in the inner detector and the rejection factor, new algorithms have been elaborate to take it into account. A larger in $\phi$ cluster is also used to reconstruct the energy of converted photons. These converted photons exhibit also a correlation between the lateral leakage measured in the first compartment and the fraction of energy deposited in this first layer. This helps also to improve the rejection factor. The $\pi^{0}$ rejection factor which is $2.95 \pm 0.06$, reaches $3.21 \pm 0.07$ when taking into account the conversion position for a photon efficiency of $90 \%$. It can be improve up to $3.31 \pm 0.07$ by using the lateral leakage correlation with the energy ratio in the first layer.

This analysis has been checked on specific testbeam data [5], obtained by inserting some material in the beam line upstream of a bending magnet, to cause the incoming electron to emit hard bremsstrahlung photons. By selecting events with the appropriate kinematics, it was possible to mimick a $\pi^{0}$ into to two photons. The agreement between simulation and testbeam data is satisfactory, as it can be seen on Fig. 2 . The rejection factor experimentally measured is $3.54 \pm 0.12$, for a efficiency of $90 \pm 1 \%$, which can be compared with $3.48 \pm 0.10$ obtained in simulation for unconverted photons.

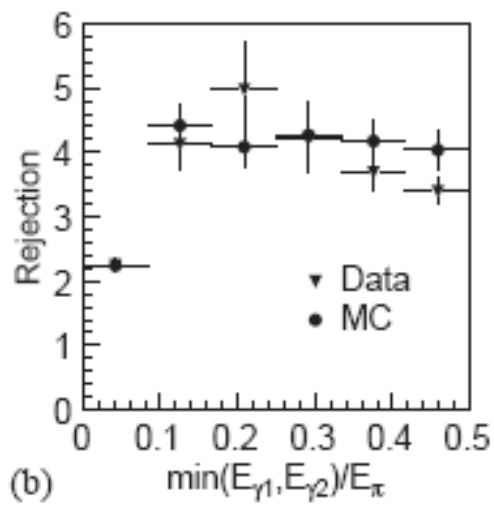

Fig. 2. $\pi^{0}$ rejection calculated in bins of the fraction of the $\pi^{0}$ energy carried by the least energetic photon, for data and simulation [5]. 


\section{Electron/pion separation}

The efficient tagging of low energy electrons is an important tool for B-physics, as well as a complementary method to b-tagging. Separating low energy electrons from pions by analyzing the energy deposits in the calorimeter alone is not an easy task, since these electrons are within are near to jets. Instead the traking information must be used to seed the calorimeter clustering. The strategy consists of several steps. First, tracks with $p_{T}>2 \mathrm{GeV} / \mathrm{c}$ are found in the inner detector and then we look the electromagnetic calorimeter regions hit by the tracks. By combining various shower shape estimators, et $E / p$ value and the information from the TRT it is possible to get the pion rejection versus the electron identification efficiency.

In the case of a $\mathrm{J} / \psi$ sample, a rejection factor of pion tracks 1000 is achieved for an electron identification efficiency of $80 \%$. This allows for the reconstruction of the $\mathrm{J} / \psi$ events with a signal to background ratio around 2. Electrons coming from $\mathrm{W} \mathrm{H} \rightarrow \mathrm{b}$ b-bar events are located inside jets. For a $80 \%$ electron identification efficiency, rejection of pion tracks from background sample is about 250 . This soft electron identification could then be used for b-tagging purpose, and has been shown to be a complementary method to standard vertex-based tagging, despite the small branching ratio.

A good e/ $\pi$ separation capability is important up to $200 \mathrm{GeV} / \mathrm{c}$. The separation of electron and pion signals in the barrel electromagnetic calorimeter was studied using electron and pion test-beam in the momemtum range from 20 to $180 \mathrm{GeV} / \mathrm{c}$. The electromagnetic shower generated by the electrons is expected to be fully contained in the electromagnetic calorimeter, while the hadronic shower is expected to leak at the back of the electromagnetic calorimeter into the hadronic calorimeter. However, a fraction of the hadronic showers may be fully contained witin the electromagnetic calorimeter creating a potential for particle misidentification. It has been found that the spread of the shower through the compartments of the electromagnetic calorimeter described as the number of hit cells in these layers provide a significant discriminative power. The analysis showed a good e/ $\pi$ separation capability in the whole studied momentum range. Using information from the liquid argon electromagnetic calorimeter only and allowing $90 \%$ electron identification efficiency, we can achieve pion fake rate of $3.2 \pm 0.2 \%$ with simple cuts at $20 \mathrm{GeV} / \mathrm{c}$. Assuming track momentum is well measured with the inner detector, the pion fake rates can be reduced to $0.50 \pm 0.07 \%$ at $20 \mathrm{GeV} / \mathrm{c}$ while still maintaining $90 \%$ electron identification efficiency. Further significant improvements were obtained by using Neural Network analysis method. With such a method we can achieve a pion fake rate of $0.34 \pm 0.08 \%$ at $20 \mathrm{GeV} / \mathrm{c}$ with $90 \%$ electron identification efficiency.

\section{Electron/jet separation}

The identification of isolated electrons with $p_{T}>20 \mathrm{GeV} / \mathrm{c}$ will be essential for the physics searched at the LHC. A challenging task is to identify electrons in the presence of a huge QCD jets background, which is $\sim 10^{5}$ times higher, as in the case of $\mathrm{W}$ and top decays. To separate electrons from jets, cuts were developed to maintain a reasonable electron identification efficiency even with pile-up at high luminosity, while removing a high fraction of jet events. The cuts include Level 1 and High Level Trigger cuts, shower shape and isolation cuts in the calorimeter, cuts on tracking inner detector (ID), cuts on ID-Calo matching in position and energy, and transition radiation cuts. The effect of applying all these cuts on e after the other to a single electron sample and an inclusive jet sample are shown in table 1. The last line here corresponds to all cuts applied. An electron identification efficiency of about $73 \%$ is obtained while a jet rejection of about $2.2 \times 10^{5}$ is achieved. Most of the raimaining candidates in the jet sample, being electrons from conversions, the application of the conversion identification algorithm allows the identification of a pure electron inclusive sample with a jet rejection around $10^{6}$.

\section{Conclusion}

The particle identification capability relying on the ATLAS liquid argon electromagnetic calorimeter evaluated with simulation and checked with testbeam data give us confidence that the ATLAS 
detector will meet the very challenging specifications for an multi-purpose LHC detector.

In the year 2004, ATLAS collaboration has been involved in a huge combined test-beam effort. A complete slice of the central region including inner detectors, calorimeters and muon chambers has been tested. A lot a data (90 million of events, $4.6 \mathrm{~Tb}$ ) has been collected. The analysis of these data is ongoing. It will help us to check the full power of the ATLAS detector for detailed particle identification and measurement.
Table 1. Electron identification efficiency $\varepsilon_{\mathrm{e}}$ and jet rejection of the offline analysis at low luminosity.

\begin{tabular}{lcc}
\hline & $\varepsilon_{\mathrm{e}}($ in $\%)$ & jet rejection $\left(\times 10^{3}\right)$ \\
\hline Calo & $91.5 \pm 0.4$ & $3.01 \pm 0.1$ \\
ID & $87.4 \pm 0.5$ & $35.9 \pm 2.5$ \\
ID-Calo & $82.2 \pm 0.6$ & $103 \pm 12$ \\
TRT & $79.0 \pm 0.6$ & $222 \pm 38$ \\
\hline
\end{tabular}

\section{References}

[1] The ATLAS collaboration, Calorimeter Performance Technical Design Report, CERN/LHCC/96-40, 1996.

[2] The ATLAS collaboration, Liquid Argon Calorimeter Technical Design Report, CERN/LHCC/96-41, 1996.

[3] B Aubert, et al, Nucl. Instr. and Meth. A 500 (2003), 178.

[4] B Aubert, et al, Nucl. Instr. and Meth. A 500 (2003), 202.

[5] J. Colas, et al, Nucl. Instr. and Meth. A 550 (2005), 96.

[6] The ATLAS collaboration, Detector and Physics Performance Technical Design Report, CERN/LHCC/99-14, 1999. 